Génét. Sél. Evol., 1988, 20 (4), 441-460

\title{
La variation géographique du polymorphisme enzymatique dans les populations de Cepaea nemoralis des Pyrénées françaises
}

\author{
Marta VALDEZ *, I. KASSEM ** et M. LAMOTTE ** \\ * Université Paris-Sud, Laboratoire d'Amélioration des Plantes, \\ 91405 Orsuy Cedex, France \\ ** Ecole Normale Supérieure, Laboratoire de Zoologie, \\ 46, Rue d'Ulm, 75230 Paris Cedex 05, France
}

\begin{abstract}
Résumé
La variation des fréquences alléliques à trois locus commandant des caractères de coloration de la coquille et à neuf locus enzymatiques a été étudiée dans 69 populations de Cepaea nemoralis provenant de plusieurs vallées des Pyrénées françaises. Des modes différents de variation génétique semblent exister pour les fréquences des divers gènes des locus enzymatiques et différents aussi de ceux des fréquences des caractères phénotypiques de la coquille. Les résultats suggèrent que plusieurs facteurs, sélectifs et fortuits, interviennent dans la différenciation protéinique de ces populations, facteurs dont l'importance relative dépend des conditions locales - notamment la sévérité du climat et l'intensité de l'activité humaine - ainsi que de l'histoire évolutive de ces populations.
\end{abstract}

Mots clés : Cepaea, variation enzymatique, polymorphisme, effet de fondation, sélection climatique.

\author{
Summary \\ Geographic variation of enzyme polymorphism \\ in populations of Cepaea nemoralis from the French Pyrénées
}

The variation of allele frequencies at three shell loci and at nine enzyme loci has been investigated in 69 populations of Cepaea nemoralis in several valleys of the French Pyrénées. Different modes of genetic variation appear to exist for the frequencies of various enzyme loci, which are also different from those of the phenotypic characters frequencies of shell. Results show that several selective and random factors are involved in the protein differentiation of these populations. The relative importance of these factors varies with local conditions, notably the degree of climate severity and the intensity of human activity, as well as with the evolutionary history of these populations.

Key words : Cepaea, enzymatic variation, polymorphism, founder effect, climatic selection. 


\section{Introduction}

L'existence d'un remarquable polymorphisme de la coquille dans presque toutes les populations naturelles de Cepaea nemoralis (L.) a suscité de nombreuses recherches en vue de déterminer les facteurs qui en sont responsables. LAMOTTE $(1959,1966)$, puis JoNEs et al. (1977) ont publié sur ce problème des mises au point assez complètes, d'où il ressort que de nombreux facteurs, sélectifs ou fortuits, interviennent dans le maintien du polymorphisme et que leur importance relative varie selon les conditions particulières des diverses populations.

Dans la région pyrénéenne, les populations de $C$. nemoralis sont caractérisées par des fréquences phénotypiques originales, par la présence simultanée d'individus à péristome blanc et à péristome coloré, enfin par la taille très grande de la coquille. Il était intéressant d'étendre à un plus grand nombre de locus les connaissances sur la composition génétique de ces populations.

L'application des techniques électrophorétiques aux études de la génétique des populations a mis en évidence l'existence d'un polymorphisme enzymatique considérable dans les populations naturelles de nombreuses espèces (voir par exemple Pasteur. 1974).

MANwell \& BAKER (1968) sont les premiers à avoir utilisé les techniques d'électrophorèse chez $C$. nemoralis. Divers travaux ont été consacrés depuis à l'étude du polymorphisme enzymatique dans plusieurs régions de l'aire de répartition de l'espèce (Brussard, 1975 ; Johnson, 1976). Certains ont été réalisés dans les Pyrénées centrales espagnoles (Jones et al., 1980 ; Caugant et al., 1982), d'autres dans les Pyrénées françaises et espagnoles (Ochman et al., 1983 ; Valdez-Forsans, 1986). Ochman et al. (1983) ont souligné l'existence de trois groupes de populations très différenciés respectivement localisés à l'est, au centre et à l'ouest des Pyrénées, suggérant un phénomène d'area effects. Ce terme avait été défini, à propos des phénotypes de la coquille de Cepaea, comme "la constance de la fréquence d'une forme sur de vastes étendues en dépit des variations de l'habitat ". Ce phénomène, observé principalement dans plusieurs districts de basse altitude en Angleterre, a été attribué soit à l'intervention prédominante de facteurs sélectifs régionaux (CAIN \& CuRREY, 1963), soit à des effets de fondation (GoodHarT, 1963).

D'après OChMAN et al. (1983) les area effects moléculaires seraient le résultat des effets de fondation survenus après la fin de la dernière glaciation du Pléistocène ; la faible différenciation génétique actuellement observée à l'intérieur de chacun des trois groupes de populations serait due, principalement, à la dérive génétique. Cette conclusion s'appuie malheureusement sur l'analyse de faibles effectifs par population et sur un nombre limité d'échantillons par vallée.

Il semble donc intéressant d'étudier plus en détail la variabilité enzymatique dans de nombreuses populations de $C$. nemoralis à l'intérieur des vallées des Pyrénées françaises. Ces vallées présentent des variations importantes d'altitude et, par conséquent, des changements prononcés du climat et de la végétation sur une distance relativement réduite. 


\section{Matériel et méthodes}

\section{A. Sites d'étude}

Cinq secteurs représentatifs ont été plus spécialement prospectés pour l'étude du polymorphisme enzymatique chez $C$. nemoralis, et 69 populations ont été ainsi collectées. La figure 1 montre la localisation des secteurs et des populations étudiées. Les échantillons $(n \geqslant 40)$ ont été récoltés sur des surfaces inférieures aux $400 \mathrm{~m}^{2}$ correspondant à l'unité panmictique (LAmoTte, 1951).

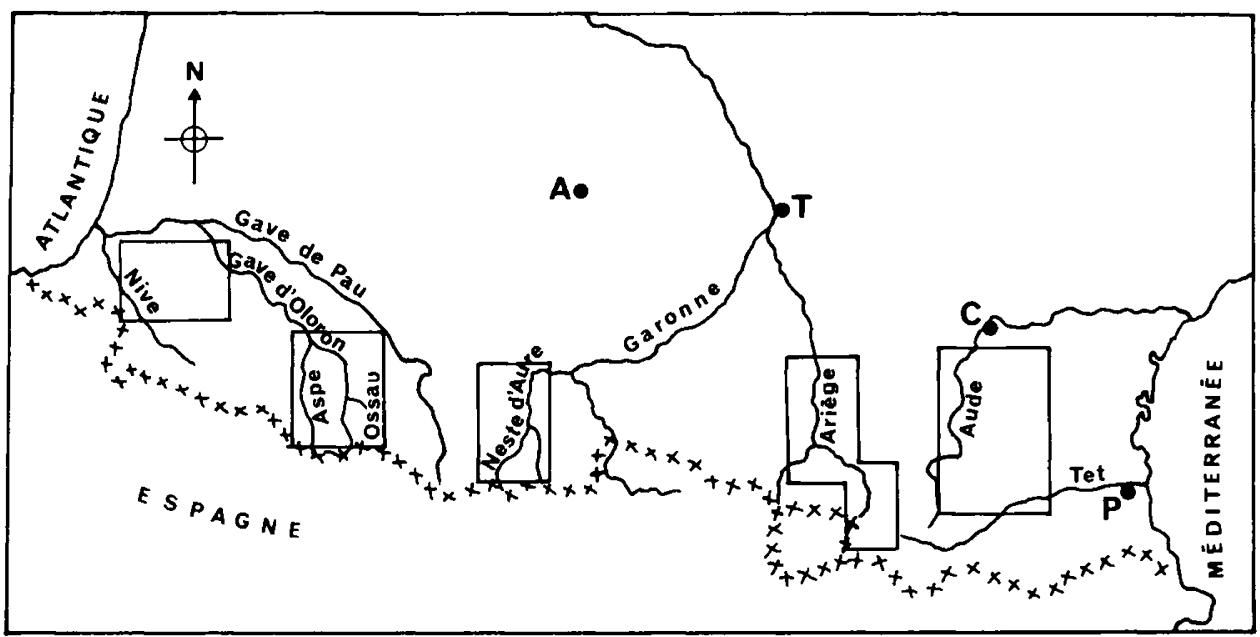

FIG. 1

Localisation des secteurs prospectés dans les Pyrénées françaises. A : Auch ; $C$ : Carcassonne ; $P$ : Perpignan ; $T$ : Toulouse.

Location of sectors prospected in the French Pyrénées.

A : Auch ; C : Carcassonne ; P : Perpignan ; T: Toulouse.

\section{B. Electrophorèse}

La technique d'extraction des protéines est celle qui a été décrite par Jones et al. (1980) et VALDEZ-Forsans (1986). Les extraits ont été soumis à une électrophorèse verticale sur gel de polyacrylamide en plaque. Le choix des enzymes et la mise au point des techniques d'électrophorèse ont été réalisés et décrits par VALDEZ-ForsANS (1986). Les locus polymorphiques étudiés concernent des estérases (trois locus : Est.-1, 2 et 3 ), la leucine-aminopeptidase (deux locus : Lap-1 et 2), la superoxyde-dismutase (un locus : Sod-1), la phosphoglucomutase (un locus: Pgm-3), la malate-déshydrogénase (un locus : $M d h-1$ ), et la phosphoglucose-isomérase (un locus : Pgi-1). Les hypothèses génétiques ont été confirmées par l'étude de neuf couples de $C$. nemoralis et de leurs descendances, examinés pour tous les systèmes polymorphiques (VAldez-Forsans, 1986). 


\section{Analyse statistique}

Les fréquences alléliques aux trois principaux locus gouvernant les caractéristiques de la coquille - couleur $(C)$, présence ou absence de bandes $(B)$ et pigmentation du péristome $(P)$ - ont été estimées à partir des proportions des génotypes récessifs. Les fréquences alléliques aux locus enzymatiques ont été estimées directement à partir des électromorphes. Une méthode de maximum de vraisemblance a été utilisée dans les cas où des allèles nuls ont été rencontrés.

L'analyse hiérarchique de WRIGHT (1978) a été employée pour estimer les variances standardisées des fréquences alléliques à plusieurs niveaux d'organisation géographique. Dans notre analyse, le modèle de différenciation dans l'ensemble de populations est décrit par une hiérarchie des indices de fixation ou variances standardisées : $F_{D R}$, populations à l'intérieur des zones altitudinales; $F_{R S}$, des zones altitudinales à l'intérieur des vallées ; $F_{S T}$, des vallées par rapport au total. Si la différenciation est due principalement aux fluctuations fortuites, les valeurs des indices de fixation aux divers niveaux d'organisation seront relativement uniformes pour tous les allèles ou locus (WRIGHT, 1978).

Pour synthétiser le modèle de variation des fréquences alléliques aux neuf locus enzymatiques et aux trois locus de la coquille, nous avons utilisé la technique d'analyse multivariée en coordonnées principales (programme ConSTEL, MEYer, 1974). Les fréquences de 27 allèles, communs à la plupart des populations étudiées, ont été " normalisées » au moyen de la transformation angulaire $(X=\operatorname{Arc} \sin \sqrt{p})$ préalablement à la réalisation de cette analyse multivariée.

La distance génétique de NEI (1972) a été calculée pour les 69 populations, prises deux à deux, à partir des fréquences alléliques aux neuf locus enzymatiques ( 35 allèles au total). Les distances ainsi obtenues ont été utilisées pour la construction d'un dendrogramme selon la méthode agrégative du groupement hiérarchique à liens simples (LEGENDRE \& LEGENDRE, 1979).

\section{Résultats}

\section{A. Polymorphisme de la coquille}

Des résultats concernant les fréquences alléliques aux trois principaux locus de la coquille dans toutes les populations de $C$. nemoralis étudiées ont été déjà présentés par VAldez-Forsans (1986) et KASSEm (1987). Les trois locus examinés sont polymorphes dans la presque totalité des populations, mais avec une prédominance de $C^{\jmath}$ (coquille jaune), $B^{0}$ (absence de bandes) et $P^{B}$ (péristome blanc).

D'une façon générale, la fréquence de $B^{\prime \prime}$ apparaît plus élevée aux altitudes supérieures $(>1200 \mathrm{~m})$ et plus diverse dans la zone basse $(<600 \mathrm{~m})$. La zone intermédiaire est au contraire caractérisée dans certaines vallées par la faible fréquence de cet allèle. 
La variation de $C^{j}$ en fonction de l'altitude dans les vallées étudiées est moins accentuée que celle de $B^{\prime \prime}$. La fréquence de $C^{\prime}$ est néanmoins plus élevée d'une part aux altitudes supérieures et d'autre part dans la partie basse. La zone intermédiaire de toutes les vallées, excepté celle de l'Aure, se caractérise au contraire par une diminution de sa fréquence.

Ces résultats sont en accord avec ceux trouvés précédemment dans la région des Pyrénées par LamotTe (1951, 1968), Arnold (1968) et Ramos (1984), qui ont suggéré un rôle important des facteurs climatiques sur les fréquences des trois locus de la coquille.

\section{B. Polymorphisme enzymatique}

La variation géographique des fréquences alléliques aux neufs locus polymorphes est présentée dans les figures 2,3 et 4 .

Estérase-1. L'allèle 100, prédominant dans les populations de la Vallée de l'Ariège et des Pyrénées orientales, montre une diminution clinale vers l'ouest, où il est absent dans les populations des Pyrénées atlantiques. Cet allèle augmente progressivement quand l'altitude diminue dans la Vallée d'Aure. L'allèle 89 , rencontré dans la Vallée d'Ossau et les Pyrénées atlantiques, augmente avec l'altitude dans la haute vallée occidentale d'Ossau.

Estérase-2. Comme pour Est.-1, il y a une forte diversité des fréquences alléliques entre secteurs. Est. $2^{(t x)}$, complètement fixé dans la Vallée d'Aure, est fortement représenté dans l'Ariège et les Pyrénées orientales; il est au contraire faiblement représenté dans la Vallée d'Ossau et les Pyrénées atlantiques où il est remplacé par l'allèle 96.

Estérase-3. L'allèle 100 prédomine dans tous les secteurs. Les fréquences de l'allèle 103 les plus élevées ont été trouvées dans la Vallée d'Ossau et les Pyrénées atlantiques. Comme dans la Vallée d'Aure, Est.- $3^{\text {(t) }}$ augmente avec l'altitude dans la Vallée d'Ossau.

Leucine-aminopeptidase-1. L'allèle 103, abondant dans les populations des Pyrénées orientales et de la Vallée de l'Ariège, est rare dans les autres secteurs où il est remplacé par l'allèle 96 qui est mieux représenté dans la haute vallée orientale d'Aure et dans la Vallée d'Ossau. La diversité génétique paraît moins élevée aux altitudes intermédiaires des vallées d'Aure et de l'Ariège.

Leucine- minopeptidase-2. Les populations des Pyrénées orientales montrent une hétérogénéi' considérable. La fréquence de l'allèle 100 augmente avec l'altitude dans les vallées de l'Ariège, d'Aure et d'Ossau. La fréquence de cet allèle, prédominant dans la Vallée de l'Ariège, baisse vers l'ouest. La diversité génétique en ce locus, relativement élevée dans tous les secteurs, augmente quand l'altitude diminue dans les vallées d'Aure et de l'Ariège, alors qu'elle augmente avec l'altitude dans la haute vallée occidentale d'Ossau. 

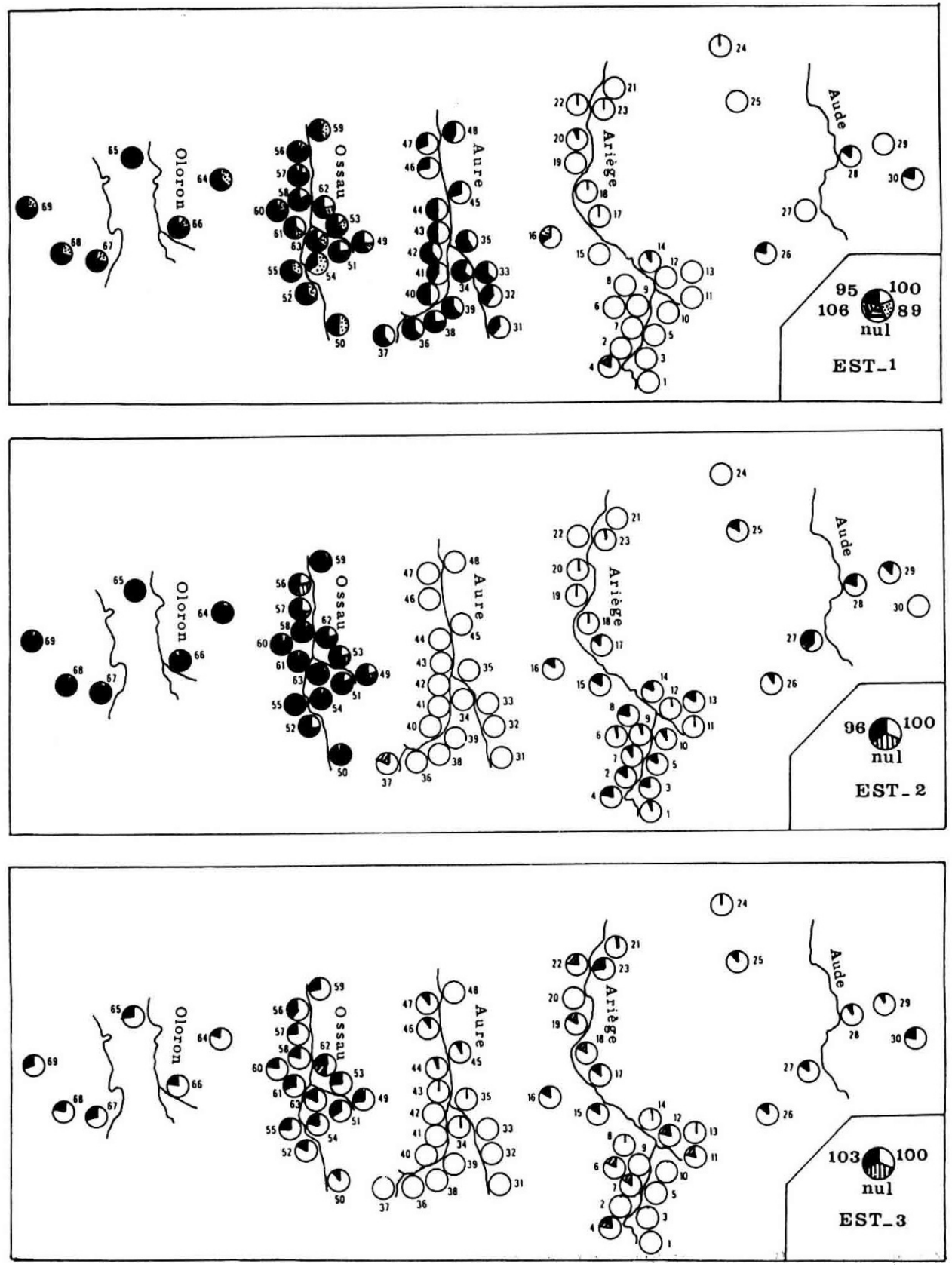

FIG. 2

Fréquences alléliques aux locus Est.-1, Est.-2 et Est.-3 dans les populations de Cepaea nemoralis des Pyrénées françaises.

Allele fequencies at the Est.-1, Est.-2 and Est.-3 loci in Cepaea nemoralis populations of the French Pyrénées. 

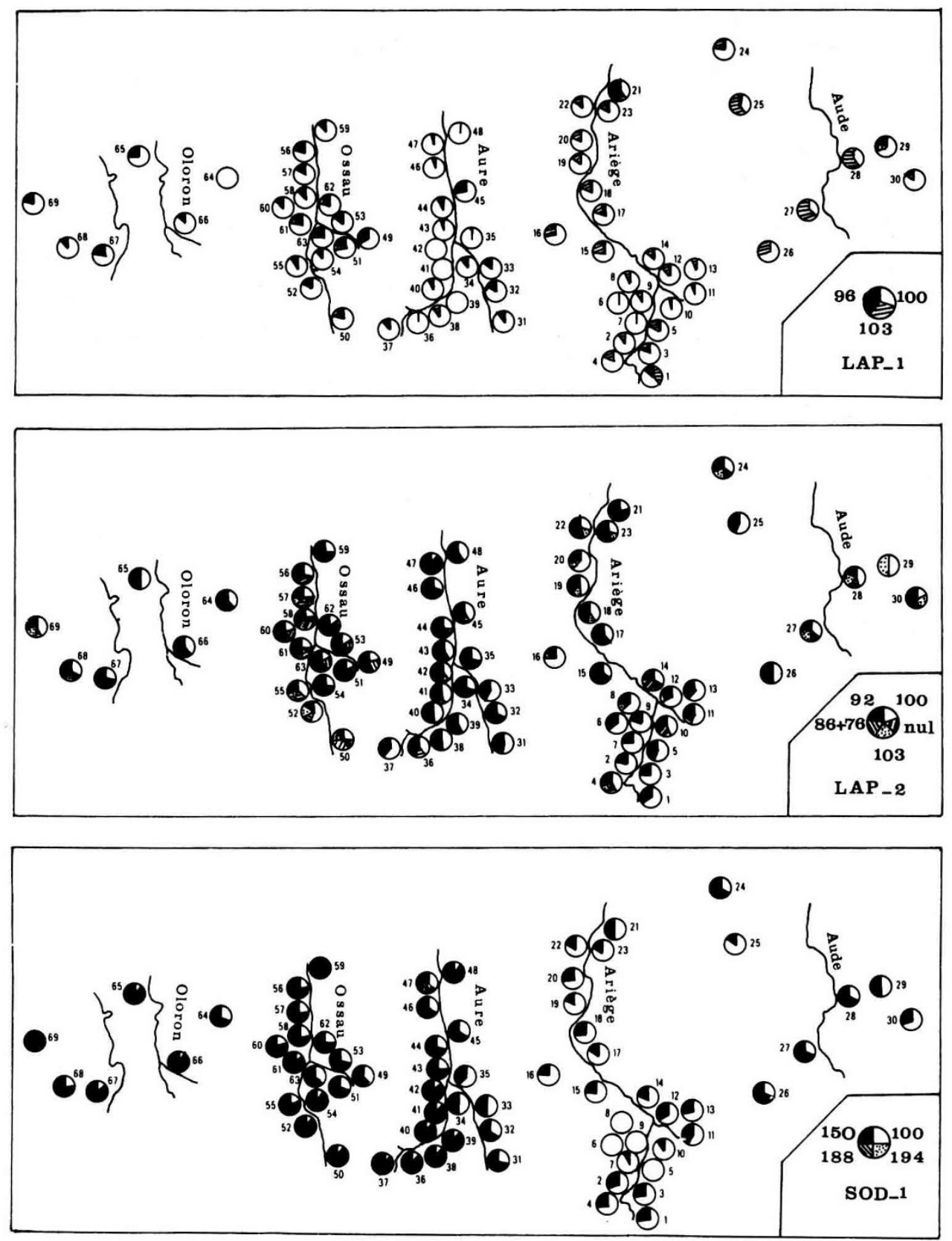

FIG. 3

Fréquences alléliques aux locus Lap-1, Lap-2 et Sod-1 dans les populations de Cepaea nemoralis des Pyrénées françaises.

Allele frequencies at the Lap-1, Lap-2 and Sod-1 loci in Cepaea nemoralis populations of the French Pyrénées. 

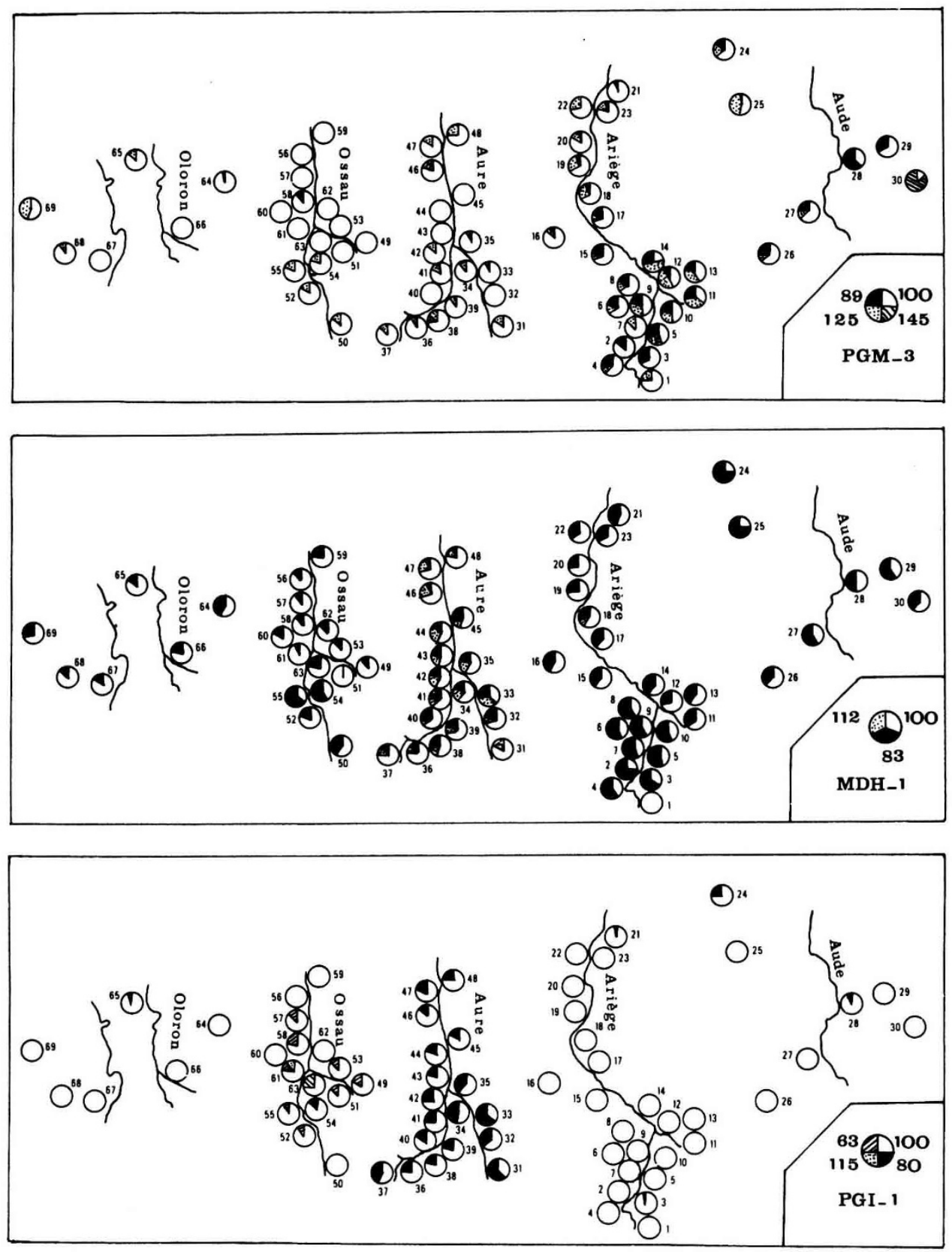

FIG. 4

Fréquences alléliques aux locus Pgm-3, Mdh-1 et Pgi-1 dans les populations de Cepaea nemoralis des Pyrénées françaises.

Allele frequencies at the Pgm-3, Mdh-1 and Pgi-1 loci in Cepaea nemoralis populations of the French Pyrénées. 
Superoxyde-dismutase-1. L'allèle 100 , majoritaire dans la Vallée de l'Ariège est au contraire minoritaire dans les Vallées d'Aure, d'Ossau et les Pyrénées atlantiques. La fréquence de cet allèle diminue progressivement quand l'altitude augmente dans la Vallée d'Aure. Le même type de variation existe dans les deux hautes vallées occidentales d'Aure et d'Ossau, où la fréquence de l'allèle 100 diminue aux altitudes supérieures. L'allèle $\operatorname{Sod}_{-1} 1^{(5)}$ est rare aux altitudes intermédiaires de la Vallée de l'Ariège.

Phosphoglucomutase-3. L'allèle 100 , fixé dans les populations de la zone basse et la haute vallée orientale d'Ossau, est fortement représenté dans la Vallée d'Aure et les Pyrénées atlantiques. Il est moins fréquent dans la Vallée de l'Ariège et les Pyrénées orientales, où la diversité génétique augmente.

Malate-déshydrogénase-1. L'allèle 100 prédomine dans tous les secteurs, mais sa fréquence augmente d'est en ouest. Elle diminue avec l'altitude dans la Vallée de l'Ariège et dans la haute vallée d'Ossau. L'allèle 112 n'a été rencontré que dans la vallée d'Aure, augmentant de ce fait la diversité génétique à ce locus dans les populations de cette vallée.

Phosphoglucose-isomérase-1 Pgi-1 $I^{(x)}$, complètement fixé dans les populations de la Vallée de l'Ariège, est fortement représenté dans celles des Pyrénées orientales et atlantiques. L'allèle 80 , rencontré dans les populations de la Vallée d'Aure, est mieux représenté dans celles de la haute vallée orientale où sa fréquence augmente quand l'altitude s'accroît. L'allèle 63 n'a été trouvé que dans les populations de la Vallée d'Ossau.

D'une manière générale, ces résultats montrent que les divers locus enzymatiques varient de façon indépendante les uns des autres et indépendamment des caractères phénotypiques. La même remarque avait déjà été faite par divers auteurs dans certains secteurs des Pyrénées (Jones et al., 1980 ; Caugant et al., 1982 ; Valdez-Forsans, 1986). L'étude des fréquences alléliques aux locus enzymatiques indique également l'existence d'une variabilité considérable, tant entre secteurs qu'à l'intérieur de chacune des vallées prospectées.

\section{Analyse statistique}

Les résultats de l'analyse hiérarchique des variances standardisées de WRIGHT (1978) sont présentés dans le tableau 1 . Les valeurs de $F_{D R}$ correspondant aux locus $C$, $B$ et $P$ apparaissent très uniformes, ce qui signifie que la variabilité observée à l'intérieur des zones altitudinales pourrait être en partie aléatoire. La valeur de $F_{R S}$, légèrement plus élevée pour $B^{\prime \prime}$, est en accord avec l'existence d'une variation altitudinale dans les vallées. Contrairement aux allèles $C^{\prime}$ et $B^{\prime \prime}$, l'indice $F_{S T}$ pour $P^{B}$ montre une forte valeur qui reflète la grande différence entre régions.

Pour les locus enzymatiques, les valeurs des divers indices montrent une certaine hétérogénéité : $F_{D R}$ varie de 0,026 à $0,113, F_{R S}$ de 0,004 à $0,082, F_{S T}$ de 0,030 à 0,663 . Le test d'homogénéité des variances de Bartlett (SoKAL \& RoHLF, 1969) a montré la signification de l'hétérogénéité de ces valeurs; $F_{D R}: \chi_{8}^{2}=37,4, P<0,001 ; F_{R S}$ : $\chi_{s}^{2}=26,9, P<0,001 ; F_{s T}: \chi_{8}^{2}=20,4, P<0,01$. D’une façon générale, ces résultats confirment l'existence des différences génétiques relatives à plusieurs des locus enzymatiques et aux locus $B$ et $P$ entre les diverses zones altitudinales, ainsi qu'entre les vallées. Conjointement, l'hétérogénéité des valeurs des indices de fixation aux trois 
niveaux d'organisation géographique suggère qu'il n'y a pas une cause commune de différenciation pour les divers locus examinés et que la variation observée ne peut donc pas être expliquée seulement par des phénomènes aléatoires de dérive génétique.

\section{TABLEAU 1}

Valeurs des indices de fixation de Wright (DR: populations à l'intérieur des zones;

RS : zones à l'intérieur des vallées; ST: vallées par rapport au total; DT : populations par rapport au total) pour les fréquences alléliques chez $C$. nemoralis dans les Pyrénées françaises

Values of Wright's hierarchical F-statistics for allele frequencies of $\mathrm{C}$. nemoralis

in the French Pyrénées (DR : populations within zones; RS : zones within valleys;

$\mathrm{ST}$ : valleys within total region sampled; DT : populations within total region sampled)

\begin{tabular}{|c|c|c|c|c|}
\hline Locus & $F_{D R}$ & $F_{R S}$ & $F_{S T}$ & $F_{D T}$ \\
\hline$C^{\prime}$. & 0,138 & 0,001 & 0,038 & 0,171 \\
\hline$\ldots \ldots \ldots$ & 0,129 & 0,010 & 0,025 & 0,159 \\
\hline$\ldots \ldots \ldots$ & 0.150 & 0,001 & 0,274 & 0,383 \\
\hline$\ldots \ldots$ & 0.085 & 0,024 & 0,062 & 0,162 \\
\hline$\ldots \ldots$ & 0,087 & 0,053 & 0,089 & 0,212 \\
\hline$\ldots \ldots$ & 0,026 & 0,082 & 0,246 & 0.326 \\
\hline$\ldots \ldots \ldots$ & 0,113 & 0,010 & 0,057 & 0,172 \\
\hline$\ldots \ldots \ldots$ & 0,085 & 0,041 & 0,030 & 0,149 \\
\hline Est. $-I$ & 0,048 & 0.042 & 0,428 & 0,479 \\
\hline Est.-2 & 0,088 & 0,004 & 0,663 & 0.694 \\
\hline Est. -3 & 0.045 & 0.011 & 0,100 & 0,150 \\
\hline Pgi-l. . & 0.047 & 0,080 & 0,129 & 0,239 \\
\hline
\end{tabular}

Quatre facteurs ont été extraits par l'analyse multivariée en coordonnées principales. Ces quatre facteurs résument au total $52,7 \%$ des informations apportées par les 27 variables (allèles) d'origine.

La projection des points représentatifs des populations sur les plans factoriels I-II et I-III est présentée dans les figures 5 et 6 . La matrice des similarités entre les variables et les facteurs extraits est donnée dans le tableau 2. On voit que le facteur I présente une similarité très élevée avec un grand nombre d'allèles : Pgm- $3^{\prime \prime(x)}$, Sod-1 $1^{15 t \prime}$,

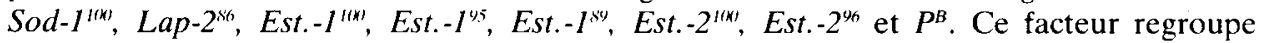
l'ensemble des populations en trois nuages bien distincts (fig. 5). Le premier concerne les populations des Pyrénées orientales et de la Vallée de l'Ariège, le deuxième regroupe celles de la Vallée d'Aure et le troisième rassemble les populations de la Vallée d'Ossau et celles des Pyrénées atlantiques.

Comme le facteur I, le facteur II traduit également la variabilité entre régions, distinguant spécialement les populations de la Vallée d'Aure de celles des autres secteurs. Il présente une similarité élevée avec plusieurs allèles des locus Pgi-l, Mdh-l, Pgm-3, Est.-1, Est. -2 et Est.-3. 


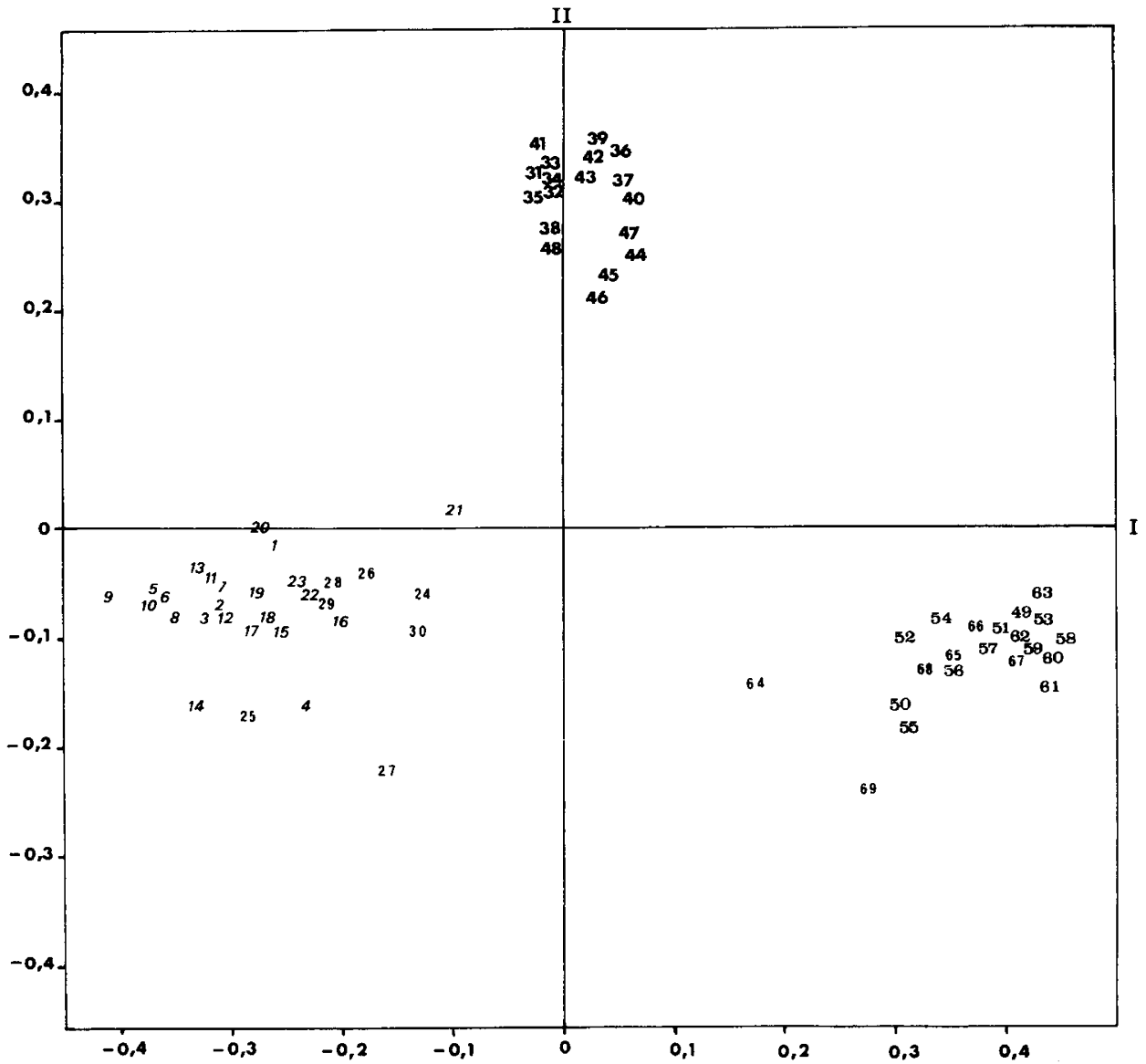

FIG. 5

Projection des échantillons de Cepaea nemoralis des divers secteurs étudiés dans l'espace des facteurs 1 et II.

1.23: pop. Vallée de l'Ariège; 24-30: pop. Pyrénées Orientales; $31-48$ : pop. Vallée d'Aure; 49-69: pop. Vallée d'Ossau et Pyrénées Atlantiques.

Projection of the Cepaea nemoralis samples on the space of factorial axes I and II.

1-23: pop. Vallée de l'Ariège; 24-30: pop. Pyrénées Orientales; $31-48$ : pop. Vallée d'Aure; 49-69: pop. Vallée d'Ossau and Pyrénées Atlantiques. 


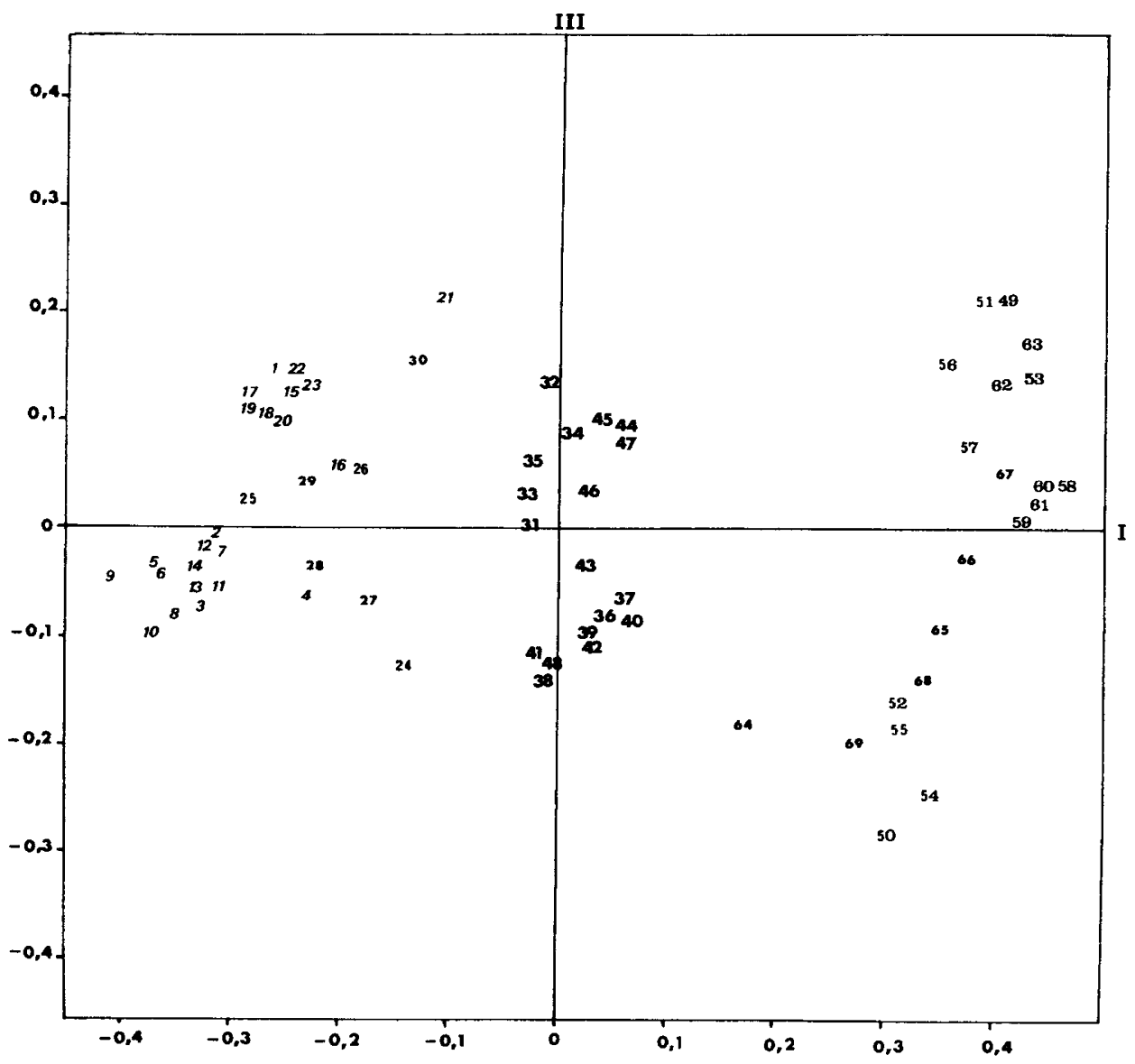

Fig. 6

Projection des échantillons de Cepaea nemoralis des divers secteurs étudiés dans l'espace des facteurs I et III.

1-23: pop. Vallée de l'Ariège; 24-30: pop. Pyrénées Orientales; $31-48$ : pop. Vallée d'Aure; 49-69: pop. Vallée d'Ossau et Pyrénées Atlantiques.

Projection of the Cepaea nemoralis samples on the space of factorial axes I and III.

1.23: pop. Vallée de l'Ariège ; 24-30: pop. Pyrénées Orientales; $31-48$ : pop. Vallée d'Aure; 49-69: pop. Vallée d'Ossau and Pyrénées Atlantiques. 
TABLEAU 2

Matrice des similarités entre variables et facteurs

Matrix of similarities between variables and factors

\begin{tabular}{|c|c|c|c|}
\hline Variables & Facteur I & Facteur II & Facteur III \\
\hline$P g i-1^{\prime(x)}$ & $-0,393$ & $-0,591$ & $-0,036$ \\
\hline$P g i-1^{80} \ldots \ldots \ldots$ & 0,154 & 0,850 & $-0,105$ \\
\hline$\ldots \ldots \ldots$ & 0,579 & 0,386 & 0,208 \\
\hline$\ldots \ldots$ & 0,508 & 0,091 & 0,188 \\
\hline$M_{d h-I^{83}} \ldots \ldots \ldots$ & $-0,558$ & $-0,429$ & $-0,266$ \\
\hline $\mathrm{Pgm} 3^{125} \ldots \ldots \ldots$ & $-0,417$ & $-0,293$ & $-0,268$ \\
\hline$\ldots \ldots$ & 0,717 & 0,397 & 0,166 \\
\hline$\ldots \ldots \ldots$ & $-0,523$ & $-0,461$ & $-0,280$ \\
\hline$\ldots \ldots \ldots$ & 0,648 & 0,188 & $-0,161$ \\
\hline$\ldots \ldots \ldots$ & $-0,717$ & $-0,199$ & 0,086 \\
\hline$\ldots \ldots \ldots$ & $-0,097$ & $-0,278$ & 0,036 \\
\hline Lap-2 $2^{100} \ldots \ldots \ldots$ & $-0,291$ & $-0,121$ & $-0,396$ \\
\hline Lap-2 $2^{92} \ldots \ldots \ldots$ & 0,233 & 0,288 & 0,343 \\
\hline$\ldots \ldots \ldots$ & 0,610 & 0,118 & 0,178 \\
\hline$\ldots \ldots \ldots$ & 0,016 & $-0,263$ & $-0,342$ \\
\hline Lap-1 $I^{100} \ldots \ldots \ldots$ & 0,038 & 0,220 & $-0,368$ \\
\hline Lap $-I^{96}$ & 0,395 & 0,010 & 0,351 \\
\hline Est. $-_{1}^{100} \ldots \ldots$ & $-0,741$ & $-0,343$ & $-0,039$ \\
\hline Est. $-^{95} \ldots \ldots \ldots$ & 0,704 & 0,280 & 0,037 \\
\hline Est. $-1^{89}$ & 0,634 & $-0,473$ & 0,091 \\
\hline Est. $-2^{100}$ & $-0,606$ & 0,499 & $-0,036$ \\
\hline Est. $-2^{96}$ & 0,733 & $-0,473$ & 0,091 \\
\hline$\ldots \ldots$ & 0,581 & $-0,486$ & 0,117 \\
\hline Est. $-3^{100}$ & $-0,263$ & 0,657 & $-0,111$ \\
\hline$C^{J} \ldots$ & $-0,188$ & $-0,105$ & 0,211 \\
\hline 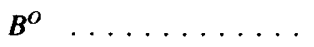 & 0,099 & 0,003 & 0,062 \\
\hline$P^{B} \ldots \ldots \ldots \ldots$ & $-0,509$ & 0,023 & $-0,218$ \\
\hline
\end{tabular}

Le facteur III divise en deux groupes les populations de chacun des trois nuages séparés par le facteur I (fig. 6). Ce troisième facteur distingue deux zones dans chaque vallée, reflétant les différences liées à l'altitude. La Vallée de l'Ariège est séparée en deux parties, au-dessus et au-dessous de $600 \mathrm{~m}$ environ, à l'exception de la population 1, isolée de l'Ariège par le Col de Puymorens. Il distingue également, dans les vallées d'Aure et d'Ossau, d'une part les populations qui proviennent des basses altitudes et de la haute vallée orientale, d'autre part celles de la haute vallée occidentale. Ce facteur présente une similarité élevée avec les fréquences des allèles 100,92 et nul de Lap-2, 100 et 96 de Lap-1. 


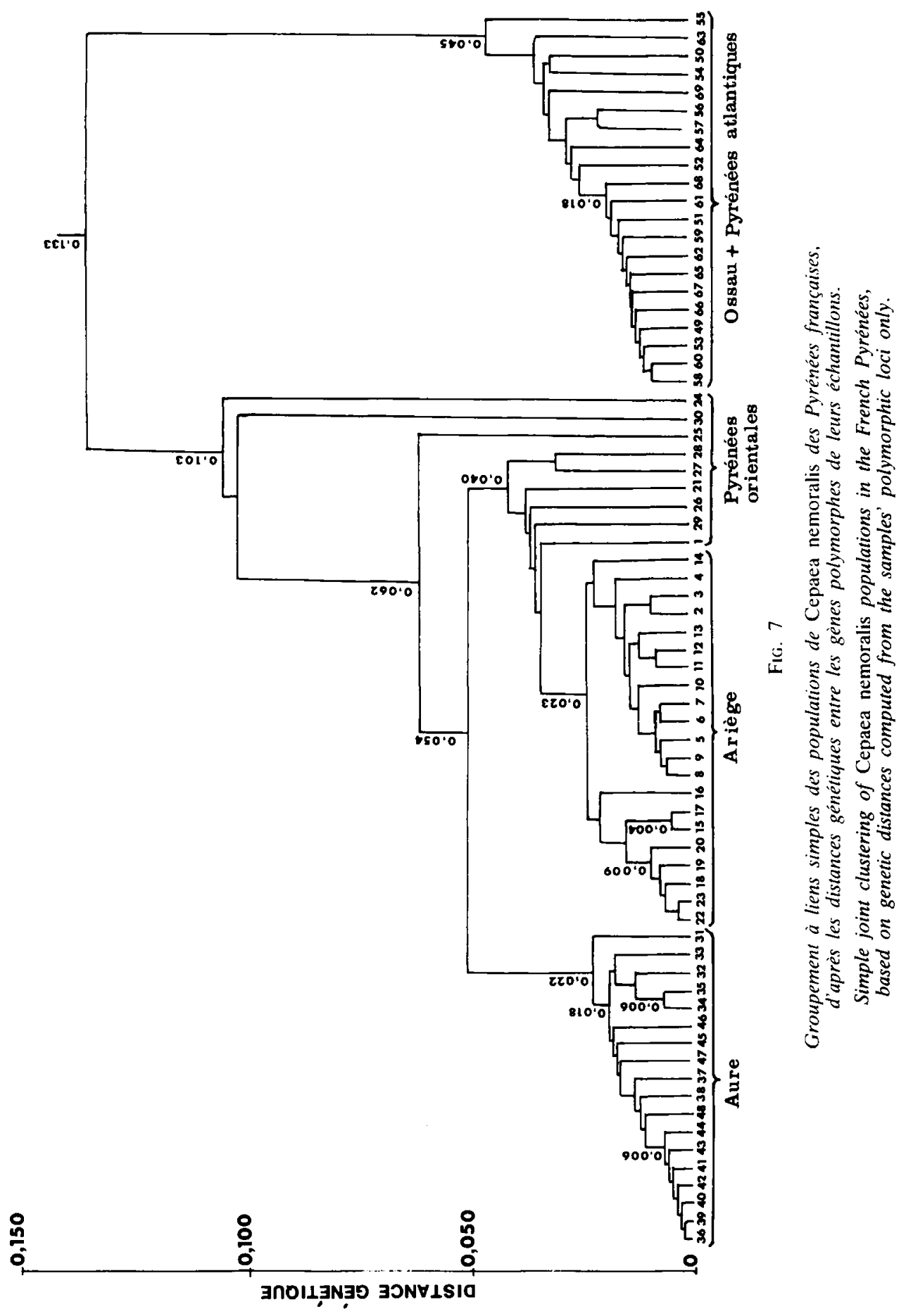


Les résultats obtenus à partir de cette analyse multivariée apparaissent donc cohérents avec les observations faites sur les variations géographique et altitudinale des fréquences alléliques. Les facteurs I et II, par suite des fortes différences entre vallées, n'expliquent que la variabilité géographique entre elles. Le facteur III, en revanche, reflète dans chacune des trois vallées la variabilité interne liée à l'altitude.

La figure 7 présente le dendrogramme réalisé à partir des distances génétiques de Nei entre les 69 populations examinées. Ces distances sont d'une manière générale élevées, du fait de la forte variabilité des fréquences alléliques des locus examinés. La signification biologique attribuée à $D$ (nombre moyen de substitutions d'allèles fixées par locus) n'a évidemment pas de sens dans notre étude, car les locus examinés ne constituent pas un échantillon représentatif de la structure du génome. Néanmoins, les estimations ainsi obtenues peuvent être utilisées comme descripteurs de la différenciation évolutive des populations.

Un écart génétique important existe entre les populations des secteurs étudiés : les distances génétiques entre les populations de la Vallée d'Aure et celles de l'Ariège sont toujours supérieures à 0,040 et les populations de la Vallée d'Ossau et des Pyrénées atlantiques montrent avec celles des autres secteurs une divergence génétique plus forte encore (souvent supérieure à 0,133 ).

Dans la Vallée de l'Ariège deux groupes de populations se différencient. Le premier correspond aux populations de basse altitude $(15,16,17,18,19,20,22,23)$. Le deuxième concerne les populations réparties dans les zones intermédiaires et de haute altitude ; à l'intérieur de ce dernier groupe, plusieurs sous-groupes se distinguent, correspondant aux populations voisines dans les diverses zones altitudinales. Les populations 1 et 21 , localisées respectivement en haute et basse altitude, se montrent les plus originales de la vallée. Ceci confirme les types principaux de variation enzymatique observés dans cette vallée (fig. 2 à 4 ).

Les populations de la Vallée d'Aure présentent entre elles une différenciation moindre que celles de la Vallée de l'Ariège. Néanmoins, deux groupes de populations se distinguent : 1) les populations de la haute vallée orientale (31 à 35), dont les distances moyennes avec les autres populations sont plutôt élevées ; 2) les populations de la haute vallée occidentale et des zones de moyenne et basse altitude (36 à 48). La différenciation observée pour certains des locus enzymatiques dans le groupe de populations de la haute vallée orientale d'Aure (fig. 2 à 4) serait donc également confirmée par cette analyse.

Le dendrogramme (fig. 7) montre aussi l'analogie des populations des Pyrénées atlantiques avec celles de la vallée voisine d'Ossau.

Dans la Vallée d'Ossau, les populations de la haute vallée occidentale gardent toujours une distance élevée par rapport aux autres populations de la vallée. En revanche, les populations de la branche orientale montrent une certaine similarité biochimique avec les populations de basse altitude. Les populations 49, 51, 53, 62 de la branche orientale constituent un groupe relativement homogène au sein duquel la distance génétique ne dépasse pas 0,026 .

Plusieurs points se dégagent de cette analyse :

a) une divergence génétique importante semble exister entre les populations des vallées étudiées, divergence qui traduit probablement l'absence totale de contacts entre ces vallées depuis de longues périodes et donc le maintien de l'identité génétique initiale de chacune des régions; 
b) il y a une certaine similarité biochimique entre les populations des Pyrénées orientales et celles de la Vallée de l'Ariège. Une similarité plus forte encore apparaît entre les populations des Pyrénées atlantiques et celles de la Vallée d'Ossau ;

c) la divergence génétique entre les populations de la Vallée de l'Ariège apparaît moins progressive et plus fortement liée à la distance géographique et à la variation altitudinale que la divergence observée entre les populations des autres vallées.

\section{Discussion et conclusions}

\section{A. Concordance entre le polymorphisme de la coquille et le polymorphisme enzymatique}

L'étude des fréquences alléliques des trois principaux locus de la coquille $(C, B$ et $P$ ) et des divers locus enzymatiques examinés dans ce travail a fait apparaître une divergence de leurs modèles de variation. Des résultats semblables ont été obtenus dans d'autres secteurs des Pyrénées par Jones et al. (1980) et Caugant et al. (1982) dont l'étude de six locus enzymatiques a montré l'absence de concordance avec la variation observée aux locus $B, C, U$ et $P$ de la coquille.

Ayala (1982) indique que, chez certaines espèces animales et chez l'homme, l'évolution des gènes de structure codant pour des protéines solubles révélées par la technique d'électrophorèse paraît indépendante de celles des gènes qui déterminent les caractéristiques de l'exophénotype. Pour tenter d'expliquer ce paradoxe, cet auteur a avancé l'hypothèse que l'évolution des caractères morphologiques proviendrait fondamentalement des changements dans les gènes de régulation et que, par conséquent, l'évolution macroscopique ne se ferait pas nécessairement à la même vitesse que celle des gènes structuraux.

Ce seraient donc des gènes de régulation qui interviendraient dans la détermination des caractéristiques de la coquille chez $C$. nemoralis, et la divergence observée par rapport aux polymorphismes enzymatiques s'expliquerait par une vitesse d'évolution différente. Des études à l'échelle moléculaire s'avèrent donc nécessaires pour essayer de comprendre le fonctionnement des gènes qui sont responsables des caractéristiques de la coquille d'une part, la relation qui existe entre ceux-ci et les gènes qui codent pour des enzymes d'autre part.

\section{B. Le rôle des divers facteurs dans la différenciation enzymatique des populations}

Les populations de $C$. nemoralis sont souvent installées dans des milieux instables et transitoires, où les extinctions et recolonisations par un petit nombre d'individus interviennent fréquemment (LAMOTTE, 1951). L'importance des fluctuations fortuites dans la différenciation génétique de ce type de populations a été soulignée par WrighT (1978). Toute interprétation de la variation du polymorphisme de $C$. nemoralis doit donc prendre en considération les événements démographiques de type historique. Or le modèle qui concerne l'influence de ce type d'événements prédit une diversification en apparence inexplicable affectant les fréquences alléliques dans les différentes populations (SLATKIN, 1977 ; JoNes et al., 1980). 
De fait, la plupart des locus enzymatiques présentent des modes de variation altitudinale qui diffèrent dans les trois vallées prospectées. Il semblerait donc qu'un facteur historique soit impliqué dans la différenciation génétique de ces populations. Deux locus cependant feraient exception : Lap-2 montre le même type de variation dans la Vallée de l'Ariège et dans la Vallée d'Aure, où la fréquence de l'allèle 100 augmente avec l'altitude, et Lap-1 dont on observe une diminution des fréquences des allèles minoritaires aux altitudes moyennes dans les trois vallées, où prédomine au contraire l'allèle 100 dans les trois groupes de populations.

L'effet des événements de type historique semble plus important dans les populations de la Vallée de l'Ariège ainsi que dans les zones de basse altitude des autres secteurs des Pyrénées où l'activité humaine est la plus forte. La très forte différenciation génétique entre les divers groupes de populations, groupes de forte similarité protéinique des populations de hautes et moyennes altitudes de la Vallée de l'Ariège, confirme ce phénomène. Comme l'a fait remarquer Lewontin (1974), des modèles en mosaïque de la variation des fréquences alléliques peuvent traduire l'effet d'accidents démographiques plutôt que l'action des facteurs sélectifs.

Dans les populations de la Vallée d'Aure, en revanche, le modèle de l'influence des événements historiques ne suffit pas pour expliquer la variation observée : quatre locus (Lap-2, Sod-1, Pgi-1 et Est.-1) montrent une transition graduelle des fréquences alléliques en fonction de l'altitude. De même, la différenciation génétique entre ces populations apparaît plus progressive et homogène (fig. 7).

L'action des facteurs climatiques semble donc plus importante sur les populations de la Vallée d'Aure. Une telle différence pourrait s'expliquer par le fait que, dans cette vallée, les biotopes sont moins perturbés par lactivité humaine que dans les autres vallées. Ainsi qu'il est normal, le rôle des facteurs climatiques dans la variation et la différenciation génétique des populations est plus facilement mis en évidence dans les sites de plus grande stabilité.

La variabilité d'un grand nombre de fréquences alléliques aux locus enzymatiques traduit une hétérogénéité considérable dans les divers secteurs. Cette différenciation est confirmée par l'analyse multidimensionnelle ainsi que par l'analyse des indices de fixation de Wright.

Dans le cadre des populations de $C$. nemoralis de la région des Pyrénées, celles des Pyrénées atlantiques et de la Vallée d'Ossau se montrent les plus originales quant aux fréquences enzymatiques : les distances génétiques qui les séparent des populations des autres secteurs sont toujours élevées. Entre ces deux secteurs existe en revanche une forte similarité enzymatique. Le même phénomène se constate, mais d'une façon moins nette, entre les populations des Pyrénées orientales et celles de la Vallée de l'Ariège. De telles ressemblances sont vraisemblablement dues aux échanges génétiques entre des populations de secteurs voisins que ne sépare aucun obstacle géographique majeur.

Au sein d'un même secteur, la plus forte variabilité des fréquences enzymatiques a été trouvée entre les populations des Pyrénées orientales. Cette divergence génétique peut s'expliquer par l'isolement plus grand de ces populations qui conduit par une sélection climatique sévère à des effets de fondation renforcés dans cette région; de fait, la température et la sécheresse d'été sont plus fortes ici que dans les autres secteurs, ce qui entraîne une raréfaction et donc un espacement des colonies de l'espèce. 
Dans les Pyrénées atlantiques, les résultats observés suggèrent au contraire l'action d'une sélection climatique tendanı̂ à homogénéiser les fréquences alléliques. La variabilité observée pourrait être imputée en partie aux différences créées par les microclimats.

D’une manière générale les vallées pyrénéennes sont étroites, ce qui accroît à l'intérieur de chacune l'importance des effets d'isolement sur la composition génétique des populations. La forte différenciation des populations de la haute vallée occidentale d'Ossau témoigne de l'importance de ces événements, car cette partie de la vallée est topographiquement séparée du reste.

De la même façon, mais à une autre échelle, l'absence relative de communications entre les populations des trois vallées de l'Ariège, d'Aure et d'Ossau, pourtant localisées toutes trois dans la partie centrale des Pyrénées françaises, joue certainement un rôle important dans la divergence de leurs populations.

Cette différenciation conduit à penseı que chaque vallée constitue une entité dont l'originalité se manifeste aussi bien par les fréquences alléliques que par les modalités de leurs variations au sein de chacune. Ceci fait évidemment penser à des effets de fondation ancestraux, comme l'ont suggéré OCHMAN et al. (1983) mais accentués et modulés par des réponses adaptatives à la diversité des conditions écologiques locales puisque l'hétérogénéité, d'un locus à l'autre, des valeurs des indices de fixation de Wright entre secteurs, montre qu'il n'existe pas une cause commune de différenciation et que celle-ci pourrait être expliquée en partie par des effets sélectifs.

Ces résultats sont en opposition avec ceux de Ochman et al. (1983), obtenus également dans les Pyrénées, et qui concluent à l'existence d'une grande uniformité des fréquences alléliques à l'intérieur de chaque vallée d'une part, et entre les vallées de la même région d'autre part. Cette opposition provient manifestement de l'échantillonnage trop restreint réalisé par ces auteurs : il est nécessaire d'analyser plus en détail ce qui se passe au sein de chaque vallée puisque, comme l'ont montré nos résultats, des différences importantes existent pour certains des locus enzymatiques examinés entre des populations très proches.

Les résultats de notre étude suggèrent donc que plusieurs facteurs, tant de type sélectif que de type aléatoire, interviennent dans la différenciation protéinique des populations étudiées de $C$. nemoralis, et que leur importance relative dépend alors des conditions locales, notamment de la sévérité du climat, et de l'histoire évolutive de ces populations.

Reçu le 26 octobre 1987. Accepté le 21 décembre 1987.

\section{Références bibliographiques}

ARNold R.W., 1968. Studies on Cepaea. 7. Climatic selection in Cepaea nemoralis (L.) in the Pyrénées. Phil. Trans. Royal Soc. London, B, 253, 549-593.

Ayala F.J., 1982. Population and evolutionary genetics : a primer. 268 p., Benjamin-Cummings, California. 
BRUSSARD P.F., 1975. Geographic variation in North American colonies of Cepaea nemoralis. Evolution, 29, 402-410.

Cain A.J., Currey J.D., 1963. The causes of area effects. Heredity, 18, 467-471.

Caugant D., Selander R.K., Jones J.S., 1982. Geographic structuring of molecular and morphological polymorphism in Pyrenean populations of the snail Cepaea nemoralis. Genetica, 57, 177-191.

Goodhart C.B., 1963. "Area effects " and non-adaptive variation between populations of Cepaea (Mollusca). Heredity, 18, 459-465.

Johnson M.S., 1976. Allozymes and area effects in Cepaea nemoralis on the western Berkshire Downs. Heredity, 36, 105-121.

Jones J.S., Leith B.H., Rawlings P., 1977. Polymorphism in Cepaea : a problem with too many solutions? Ann. Rev. Ecol. Syst., 8, 109-143.

Jones J.S., Selander R.K., Schnell G., 1980. Patterns of morphological and molecular polymorphism in the land snail Cepaea nemoralis. Biol. J. Linn. Soc., 14, 359-387.

KASSEM I., 1987. La variation géographique des polymorphismes phénotypique et enzymatique dans les populations de Cepaea nemoralis des Pyrénées françaises. Publ. Lab. Zool. E.N.S., 33, 191 p., Paris, France.

LAmotTe M., 1951. Recherches sur la structure génétique des populations naturelles de Cepaea nemoralis (L.). Bull. Biol. France (suppl. 35), 1-239.

Lamotre M., 1959. Polymorphism of natural populations of Cepaea nemoralis. Cold Spring Harbor Symp. Quant. Biol., 24, 65-86.

LamotTe M., 1966. Les facteurs de la diversité du polymorphisme dans les populations naturelles de Cepaea nemoralis (L.). Lav. Soc. Malac. Ital., 3, 33-73.

Lamorte M., 1968. Les traits généraux du polymorphisme de la coquille dans les populations naturelles de Cepaea nemoralis (Mollusques, Helicidae) des Pyrénées françaises. C.R. Acad. Sci. Paris, sér. D, 267, 1318-1321.

Legendre L., Legendre P., 1979. Ecologie numérique. Tome 2. La structure des données écologiques. 247 p., Masson, Paris.

Lewontin R.C., 1974. The genetic basis of evolutionary change. 346 p., Columbia Univ. Press, New York.

MANwell C., BAKER C.M.A., 1968. Genetic variation of isocitrate, malate and 6-phosphogluconate dehydrogenases in snails of the genus Cepaea : introgressive hybridization, polymorphism and pollution? Comp. Biochem. Physiol., 26, 195-209.

MeYer J.A., 1974. Constel : a Fortran IV program for factor and cluster analysis of mixed data. Behav. Methods Instrum., 6, 506.

Nel M., 1972. Genetic distance between populations. Amer. Natur., 106, 283-292.

Ochman H., Jones J.S., Selander R.K., 1983. Molecular area effects in Cepaea. Proc. Natl. Acad. Sci. USA, 80, 4189-4193.

Pasteur G., 1974. Génétique biochimique et populations, ou : pourquoi sommes-nous multipolymorphes ? Mém. Soc. Zool. France, 37, 473-531.

Ramos M.A., 1984. Polymorphism of Cepaea nemoralis (Gastropoda, Helicidae) in the Spanish occidental Pyrénées. Malacologia, 25 (2), 325-341.

Slatkin M., 1977. Gene flow and genetic drift in a species subject to frequent local extinctions. Theoret. Pop. Biol., 12, 253-262.

Sokal. R., Rohlf J., 1969. Biometry. 776 p., W.H. Freeman, San Francisco.

Valdez-Forsans M., 1986. Le polymorphisme enzymatique chez les Cepaea nemoralis (Moll. Pulmonés) de deux vallées pyrénéennes. Publ. Lab. Zool. E.N.S., 29, 181 p., Paris, France.

WRIGHT S., 1978. Evolution and the genetics of populations. Vot. 4. Variability within and among natural populations. 480 p., Univ. Chicago Press. 